\title{
The Association Between Triglyceride-Glucose Index, Cardio-Cerebrovascular Diseases, and Death in Korean Adults: A Longitudinal Study Based on the NHIS-HEALS Cohort
}

Joungyoun Kim

Yonsei University College of Nursing

Sang-jun Shin

Chungbuk National University

Hee-Taik Kang ( $\sim$ kanght0818@gmail.com )

Chungbuk National University College of Medicine https://orcid.org/0000-0001-8048-6247

Original investigation

Keywords: insulin resistance, triglycerides, glucose, cardiovascular diseases, cerebrovascular disorders, mortality

Posted Date: June 30th, 2021

DOl: https://doi.org/10.21203/rs.3.rs-663384/v1

License: (c) (1) This work is licensed under a Creative Commons Attribution 4.0 International License.

Read Full License 


\section{Abstract}

Background: The triglyceride-glucose (TyG) index is a reliable indicator of insulin resistance. We aimed to investigate the TyG index in relation to cardio-cerebrovascular diseases (CCVDs) and mortality.

Methods: This retrospective study included 114,603 subjects. The TyG index was categorized into four quartile groups by sex: $Q_{1},<8.249$ and $<8.063 ; Q_{2}, 8.249-<8.614$ and $8.063-<8.403 ; Q_{3}, 8.614-<$ 8.998 and $8.403-<8.752$; and $Q_{4}, \geq 8.998$ and $\geq 8.752$, in men and women, respectively. To calculate hazard ratios (HRs) and $95 \%$ confidence intervals (Cls) for the primary outcomes (CCVDs and all-cause mortality), Cox proportional hazards regression models were adopted.

Results: Compared to $Q_{1}$, fully adjusted HRs ( $95 \% \mathrm{Cls}$ ) for the primary outcomes of $Q_{2}, Q_{3}$, and $Q_{4}$ were 1.063 (0.982-1.152), 1.112 (1.026-1.206), and 1.153 (1.060-1.254) in men and 1.099 (0.986-1.226), 1.049 (0.941-1.169), and $1.069(0.960-1.190)$ in women, respectively. HRs (95\% Cls) for cardiovascular diseases (CVDs) of $Q_{2}, Q_{3}$, and $Q_{4}$ were 1.117 (0.971-1.285), 1.191 (1.036-1.369), and 1.237 (1.071$1.427)$ in men and 1.239 (1.018-1.509), 1.188 (0.976-1.446), and 1.248 (1.027-1.517) in women, respectively.

Conclusions: The elevated TyG index were was positively associated with CCVDs and all-cause mortality in men and predicted the higher risk of CVDs in both sexes.

\section{Introduction}

Cardiovascular diseases (CVDs) and cerebrovascular diseases (CbVDs), which include coronary heart disease, peripheral arterial disease, and deep vein thrombosis in addition to other diseases of the heart and brain, are the leading cause of death worldwide [1]. CVDs and CbVDs are the second and fourth most common causes of death in Korea, respectively [2]. These cardio-cerebrovascular diseases (CCVDs) are a large burden on public health and a considerable number can be avoided by early detection and active management of their risk factors such as a healthy lifestyle.

Insulin resistance plays an important role in the development of diabetes mellitus and may be closely associated with atherosclerotic CCVDs, malignant neoplasms, and mortality [3-5]. The early detection of insulin resistance is important in reducing the burden of chronic diseases. The hyperinsulinemiceuglycemic glucose clamp method is the gold standard to measure insulin resistance [6]. However, it is difficult to apply this technique to a real-world clinical setting because of its complex measurement procedure. The homeostasis model assessment of insulin resistance (HOMA-IR) is frequently used in the clinical setting but also has limited applicability because insulin levels must be measured [7]. The triglyceride-glucose (TyG) index is a novel reliable indicator to detect the early phase of insulin resistance and type 2 diabetes [8-10]. Furthermore, several studies have reported that the TyG index was superior to HOMA-IR in predicting type 2 diabetes in the Korean population [10]. In part, the dietary pattern of the 
Korean populations who consume high levels of carbohydrates and low levels of fat may contribute to the higher predictability of the TyG index.

This study aimed to examine whether the TyG index was associated with primary outcomes (CCVDs and all-cause mortality) in Korean adults, based on the Korean National Health Insurance Service (NHIS)National Health Screening (HEALS) cohort. In addition, we further investigated the association between the TyG index and each secondary outcome after stratifying the primary outcomes into CVDs, CbVDs, CCVD-related death, and all-cause death.

\section{Methods}

\section{Study population}

The NHIS-HEALS cohort included 514,794 subjects who were randomly selected from the 5.1 million examinees of the national health check-up program from January 2002 to December 2003. The age of the subjects was between 40 and 79 years at the end of December 2002. The cohort contains the lifestyle information of subjects and past medical history of diseases based on the self-reported questionnaires, diagnostic codes, and prescription information based on national insurance claim data, death information such as the main cause and date, and laboratory information from biennial national health check-up programs. The NHIS-HEALS cohort did not collect specific lipid profile such as triglycerides (TG) except for total cholesterol levels between 2002 and 2008. Thus, we should set 2009-2010 as the baseline because TG have been available since 2009.

The flowchart describes the inclusions and exclusions of this study (Figure 1). Initially, 362,285 subjects who underwent the national health check-up programs between 2009 and 2010 were included. Among the initial subjects, individuals who met one or more of the following criteria were excluded: 1) subjects who died between 2009 and $2011(n=3644) ; 2$ ) subjects who had a fasting blood glucose levels $\geq 126$ $\mathrm{mg} / \mathrm{dL}$ between 2002 and $2010(n=64,448) ; 3)$ subjects who were prescribed anti-diabetic drugs between 2002 and 2010 ( $n=9695)$; 4) subjects who were diagnosed with diabetes between 2002 and 2010 (ICD10 code: E10-E14) $(n=20,580) ; 5)$ subjects who were diagnosed with any neoplasm (ICD-10 code: C00C97 or D00-D09) ( $n=24,789)$ or CCVDs (the International Classification of Diseases [ICD]-10 code: I20-I25 for CVDs or 160-169 for CbVDs) ( $n=55,095)$ between 2002 and $2011 ; 6)$ subjects who had any history of diabetes, heart disease, CVDs, or CbVDs according to the self-reported questionnaires of the national health check-up program between 2002 and $2010(n=2,686)$; 7) subjects who were prescribed lipidlowering agents (such as statin, omega-3 fatty acids, niacin, or cholesterol sequestrants) between 2002 and $2010(n=30,615) ; 8)$ subjects who had incomplete data for the confounders $(n=6036)$; or 9$)$ subjects whose study duration was 30 days or less $(n=94)$. After these exclusions, 144,603 subjects (78,021 men and 66,582 women) were included in this study.

This research followed the 1964 Helsinki Declaration and was approved by the Institutional Review Board of Chungbuk National University Hospital (CBNUH 2021-03-003) 


\section{Definition of TyG index, cardio-cerebrovascular disease, death, and study duration}

The TyG index was calculated using the following equation: TyG $=\mathrm{Ln}(\mathrm{TG}[\mathrm{mg} / \mathrm{dL}]$ glucose $[\mathrm{mg} / \mathrm{dL}] / 2)$ $[11,12]$. Calculated TyG indices were divided into four groups by sex: $Q_{1},<8.249$ in men and $<8.063$ in women; $Q_{2}, 8.249-<8.614$ in men and $8.063-<8.403$ in women; $Q_{3}, 8.614-<8.998$ in men and 8.403 $-<8.752$ in women; and $Q_{4}, \geq 8.998$ in men and $\geq 8.752$ in women.

The primary end point of this study is to compare the occurrence rates of CCVDs and all-cause mortality by TyG quartile groups after enrollment (2009-2010). CCVDs included CVDs (I20-I25) and CbVDs (I60169) based on the ICD-10 code. CCVDs were defined when the main diagnosis (I20-125 or 160-169) was recorded at least twice in outpatients or once in hospitalized patients. As secondary outcomes, we considered the subgroup analyses for each CVD, CbVD, CCVD-related death, and all-cause deaths. In the case of deceased patients, it was classified by the cause of death recorded in the death certificate. If subjects died from fatal CCVD and the direct cause of death was recorded as CVD or CbVD on his/her death certificate, they were defined as CCVD-related deaths.

The research start date was defined as the day of the first health check-up at baseline (2009-2010). If a subject experienced CCVDs or death, the end date is the earlier date of the events. Otherwise, the end date is the last of the two following cases: the date of the last outpatient clinic visit or the last health check-up.

\section{Definition of covariates}

Risk factors that can attribute to CCVDs or deaths were controlled such as age, body mass index (BMI), systolic blood pressure (SBP), low-density lipoprotein cholesterol (LDL-C), lifestyle (cigarette smoking, alcohol consumption, and physical activity), and economic status (monthly household income). These confounding factors were surveyed at baseline (2009-2010).

BMI (unit, $\mathrm{kg} / \mathrm{m}^{2}$ ) was calculated as body mass $(\mathrm{kg})$ divided by squared height $(\mathrm{m})$. Lifestyle and household income levels were collected from self-reported questionnaires. Smoking status was classified as ever smokers (who had smoked cigarettes in the past) and never smokers (who had never smoked cigarettes). Alcohol consumption was categorized into rare (less than once a week), sometimes (once to twice a week), and often (three or more times a week). Physical activity was divided into rare (less than once a week of any kind of exercise), sometimes (individuals who did not meet the definition of rare or regular physical activity), and regular (five or more times of walking or moderate-intensity exercise a week; three or more times of vigorous-intensity a week; four or more times of walking, moderate-intensity physical activity or vigorous-intensity exercise a week). Economic status was classified into three groups, based on the self-reported monthly household income: low, 0 to $30^{\text {th }}$ percentile; middle, $31^{\text {st }}$ to $70^{\text {th }}$ percentile; and high, $71^{\text {st }}$ to $100^{\text {th }}$ percentile.

\section{Statistical analysis}


Continuous and categorical variables were presented as mean \pm standard deviations (SDs) and the number of subjects (\%), respectively. To check the group difference, analysis of variance (ANOVA) tests for continuous variables and chi-square tests for categorical variables were used. To investigate the association between the TyG index and outcomes (CVD, CbVD, and deaths), outcome-free survival rates were estimated and compared using the Kaplan-Meier method and the log-rank test. The hazard ratios (HRs) and 95\% confidence intervals (Cls) were calculated to examine the association between the TyG index and the primary outcomes (CCVDs and all-cause mortality) based on Cox proportional hazards regression models after adjusting for confounders. Three Cox proportional hazards regression models were built: 1) Model 1, age only; 2) Model 2, age, smoking status, alcohol consumption, and physical activity; 3) Model 3, BMI, SBP, LDL-C, in addition to the variables in Model 2. All p-values were two-sided and statistical significance was set as $<0.05$. The statistical analyses were performed using the statistical package SAS enterprise version 7.1 (SAS Inc., Cary, NC) and R studio version 3.3.3.

\section{Results}

In total, 144,603 subjects $(78,021$ men and 66,582 women) were included and the median study duration was 5.97 years.

Table 1 presents the baseline characteristics of the subjects according to the TyG quartile by sex. Mean age increased in men but decreased in women with the higher quartile group. In both sexes, BMI, SBP, glucose, TG, and percentage of ever smokers increased with increased quartile while the percentage of regular physical activity decreased (all p-values $<0.001$ ). Mean TyG index from $Q_{1}$ to $Q_{4}$ was $7.945,8.438$, 8.798 , and 9.359 , respectively, in men and $7.775,8.240,8.570$, and 9.086 , respectively, in women.

Figure 2 shows the estimated cumulative event incidence, based on the Kaplan-Meier's survival curve: 2A) the primary outcomes (CCVDs and all-cause mortality), 2B) CVDs, and 2C) CbVDs. Cumulative incidence of the primary outcomes was not different in men but significantly increased with the increased quartile groups in women ( $p$-value $=0.247$ in men and $<0.001$ in women) (Figure 2A). Further statistical analyses were conducted after dividing CCVDs into CVDs and CbVDs. The cumulative incidence of CVDs significantly increased with the increasing TyG index (all p-values $<0.05$ ) (Figure 2B). Cumulative incidence of CbVDs was not different in men ( $p$-value $=.0 .673$ ) but the highest in the $Q_{4}$ of women ( $p$ value $<0.010$ ) (Figure 2C).

Table 2 demonstrates the results of Cox proportional hazards regression models to examine the association between the TyG index and individual outcomes. Compared to $Q_{1}, \mathrm{HRs}(95 \% \mathrm{Cls})$ for the primary outcomes of $Q_{2}, Q_{3}$, and $Q_{4}$ were 1.063 (0.982-1.152), $1.112(1.026-1.206)$, and 1.153 (1.0601.254), respectively, in men and 1.099 (0.986-1.226), 1.049 (0.941-1.169), and 1.069 (0.960-1.190), respectively, in women after adjusting for age, smoking status, alcohol consumption, physical activity, BMI, SBP, LDL-C, and economic status. Subgroup analyses were performed to examine the association between the TyG index and individual outcomes (CVDs, CbVDs, CCVD-death, and all-cause death). Compared to $Q_{1}$, HRs (95\% Cls) for CVDs of $Q_{2}, Q_{3}$, and $Q_{4}$ were 1.117 (0.971-1.285), 1.191 (1.036- 
1.369), and 1.237 (1.071-1.427), respectively, in men and 1.239 (1.018-1.509), 1.188 (0.976-1.446), and 1.248 (1.027-1.517), respectively, in women after fully adjusting. However, the TyG index was not significantly associated with CbVDs, CCVD-related death, and all-cause death in both sexes.

\section{Discussion}

This study demonstrated that there was a positive association between the TyG index primary outcomes (CCVDs or all-cause mortality) in men, but not in women. After stratifying the primary outcomes into CVDs, CbVDs, CCVD-related death, and all-cause death, a higher TyG index predicted a higher risk of CVDs in both sexes while it was not significantly associated with CbVDs, CCVD-related death, and all-cause death.

Insulin stimulates glucose metabolism and suppresses fatty acid utilization and lipolysis as an energy source. Insulin resistance is defined as an impaired tissue response to insulin stimulation, resulting in the dysfunction of glucose and lipid metabolism [3]. These metabolic alterations lead to chronic hyperglycemia, dyslipidemia (in particular, increased free fatty acid release in adipose tissue and hepatic TG production), oxidative stress, and inflammation that induce endothelial dysfunction and atherosclerotic change. Eventually, insulin resistance increases CCVDs and premature death through these pathophysiologic mechanisms [3-5]. Thus, early detection and a more intensive management of insulin resistance are very important in reducing the likelihood of these events. Even though several methods to measure insulin resistance have been proposed, the hyperinsulinemic-euglycemic glucose clamp is considered the standard [6]. However, the hyperinsulinemic-euglycemic glucose clamp is not practical for use in real-world clinics because of complex and invasive measurements. Another reliable surrogate marker of insulin resistance is HOMA-IR. However, the disadvantage is that blood insulin levels are not routinely checked for patients without diabetes in the primary clinical setting [7].

The TyG index is newly proposed as a marker to measure insulin resistance. TG and glucose levels, which are used as variables to calculate the TyG index, are widely and commonly measured in clinics. The Korean Society of Lipid and Atherosclerosis recommends that the lipid profile (total cholesterol, TG, HDLC, and LDL-C) be checked every 4-6 years for adults to screen for dyslipidemia and CCVD risk [13]. Many guidelines recommend the annual measurement of fasting glucose levels to screen for diabetes or prediabetes $[14,15]$, while there are few recommendations for the measurement of fasting insulin levels to screen for them. Furthermore, Asian populations may consume more foods with a higher carbohydrate content than Western populations, which increases their blood TG and glucose levels [16]. Carbohydraterich diets raise the possibility of developing hypertriglyceridemia and impaired fasting glucose, which are related to the risk of CCVDs $[17,18]$. Epidemiological studies support the proposal that the TyG index is a better indicator of insulin resistance in Asian populations than $\operatorname{HOMA-IR}[10,19]$. In addition, a lot of studies report that the TyG index was more closely associated with atherosclerosis, arterial stiffness, subclinical cerebral vessel diseases, and coronary arterial diseases than HOMA-IR [20-23]. 
The primary outcome of this study is CCVDs and all-cause mortality. CCVDs are composed of CVDs (such as angina pectoris, myocardial infarctions) and CbVDs (such as non-traumatic cerebral hemorrhage, cerebral infarction, and cerebral arterial diseases, etc.). The statistical significance between the TyG index and primary outcomes are different according to statistical methods (significant only in women from the log-rank test and only in men from Cox proportional hazards model). Here we observed a very interesting result. In terms of the association between the incidence of the primary outcomes and TyG index in men, there was no significance from the log-rank test (Figure 2). However, it began to be significant after adjusting for age in the Cox proportional hazards regression model (Supplementary Table). This phenomenon is the opposite in women. That is, the log-rank test showed a significant association, but the result from the fully adjusted Cox proportional hazards regression model showed non-significance. This might be explained by the different age distribution over TyG index by sex. As the TyG quartile increases, the mean age decreases in men but increases in women. Because the log-rank test does not take any confounding factors into account, the significance in women might include the compounded effect of age and TyG index. An investigation into the conflicting relationship between age and the TyG index by sex is worthwhile. However, the elevated TyG index increased CVDs in both sexes, while it was not independently associated with individual events such as CbVDs, CCVD-related deaths, and all-cause deaths. These findings signify that the TyG index may be a good indicator in predicting CVDs such as ischemic heart diseases. Results from this study are consistent with a previous study by Park et. al. who reported that an elevated TyG index forecasted the risk of ischemic heart disease in Korean population [24]. Zhao et al. reported that the TyG index is an independent marker for cardiovascular events [25]. However, these studies have a smaller population size and did not stratify the study population according to sex. Our study supports the previous studies but more clearly clarifies the association between the TyG index and individual events. The TyG index was significantly associated with the primary outcomes (CCVDs and all-cause mortality) in men, but not in women. However, further statistical analyses demonstrated that an elevated TyG index was an independent indicator of CVD development in both sexes while there was a null association between TyG index and other outcomes (CbVDs, CCVD-related death, and all-cause death).

This study has several strengths to distinguish it from previous studies. First, the NHIS-HEALS cohort represents the entire Korean adult population. The NHIS provides reliable information on individual medical history (diagnostic code, laboratory results from national health screening program, lifestyle factors, anthropometric data, and death information based on a death certificate) and socioeconomic data (based on insurance premiums and self-reported questionnaires). The data from the NHIS-HEALS cohort were based on real-world measurements in a clinical setting. The real-world data showed the true relationship reflecting many variables and environments that we did not expect. Second, an apparently healthy population aged 40 years or older who received a national health screening examination was enrolled in this study, while patients who were diagnosed with diabetes, cancers, or CCVDs were excluded from the analysis. In addition, to control the effects of medications, such as glucose-lowering and lipidlowering drugs, individuals who had taken these drugs between 2002 and 2010 were excluded. However, individuals treated with these drugs after 2010 were not excluded in the final analyses because the aim of 
this study was to investigate the association between the initial TyG index and CCVDs or all-cause mortality regardless of medication during the follow-up period. In other words, we just tried to examine whether the elevated TyG index at baseline increases the CCVDs and all-cause mortality. These strict exclusion criteria may minimize the effects of underlying conditions on the primary outcome. Despite strict exclusion, many subjects (144,000 or more) were included in the final analyses. Third, the follow-up duration (median 5.97 years) was relatively long. In the case of apparently healthy individuals, large population size and longer study duration allowed a more accurate relationship to be explored than those from previous studies. Fourth, we widely examined the outcomes, which were related to the TyG index. The primary outcomes were stratified to several individual outcomes and sequentially investigated the relationships between them and the TyG index. In addition, many conventional risk factors including health behaviors, SBP, LDL-C, and economic status for CCVDs were adjusted in the Cox proportional hazards regression models. This conservative approach provides a reliable association between the TyG index and outcomes to minimize the function of these conventional risk factors as confounders.

There are several limitations that should be interpreted cautiously. We could not validate whether the TyG index directly correlated with insulin resistance because the NHIS-HEALS cohort did not contain information on serum insulin levels. However, the TyG index was already validated to indicate insulin resistance in previous studies [26, 27]. The outcomes such as CVDs, CbVDs, and CCVD-related deaths were not directly collected by our research team. There is the possibility of misclassification or a different definition of outcome events. The Korean health insurance system is uniquely different from other countries because all Koreans should subscribe to the NHIS and the Korean government collects and controls health information, insurance claim information, and reimbursement. For example, the NHIS assesses the coverage of insurance and determines the cost of the individual medical service, while Health Insurance Review and Assessment Service (HIRAS), another national agency, reviews the claim data and reimburses the hospital fees. Special diseases such as CCVDs, cancers, and rare diseases are more strictly monitored by these two national agencies because patients with special diseases pay $5 \%$ of their hospital fee and the NHIS pays the remaining medical cost. These strict monitoring systems minimize inaccurate diagnosis. In addition, to minimize the misclassification, we more conservatively defined the CCVDs. CCVDs were defined when the relevant diagnostic codes (120-125 or 160-169) as the main diagnostic code were recorded more than once at hospitalization or at least twice at the outpatient visit. Even if there are misclassifications, we believe that there would be very few.

In conclusion, men with an elevated TyG index were at higher risk for CCVDs or all-cause mortality. Individuals with a higher TyG index were more likely to develop CVDs in both sexes while the TyG index was not significantly associated with CbVDs, CCVD-related death, and all-cause death.

\section{Abbreviations}

ANOVA, analysis of variance; $\mathrm{BMI}$, body mass index; CbVD, cerebrovascular disease; CCVD, cardiocerebrovascular disease; $\mathrm{Cl}$, confidence interval; CVD, cardiovascular disease; HEALS, health screening; HOMA-IR, homeostasis model assessment of insulin resistance; HR, hazard ratio; ICD, international 
classification of diseases; LDL-C, low-density lipoprotein cholesterol; NHIS, national health insurance service; SBP, systolic blood pressure; SD, standard deviation; TG, triglycerides; TyG, triglycerides-glucose

\section{Declarations}

Ethics approval and consent to participate: Not applicable

Consent for publication: Not applicable

Availability of data and materials: Not applicable

Competing interests: The authors declare that they have no competing interests

Funding: Joungyoun Kim received a research grant by the National-Research-Foundation of Korea (NRF) grant funded by the Korea government (Grant No.: 2019R111A3A01059886).

Hee-Taik Kang received a research grant by the National Research Foundation of Korea (NRF) funded by the Korea government (Grant No. 2021R1G1A1006485) and a grant of the Korea Health Technology R\&D Project through the Korea Health Industry Development Institute (KHIDI), funded by the Ministry of Health \& Welfare, Republic of Korea (Grant No.: HI19C0526).

Authors' contributions: All authors made substantial contributions to the conception or design of the work. All the authors made substantial contributions to the acquisition, analysis, or interpretation of data for the work. All authors contributed to the manuscript drafting or critically revised it for important intellectual content and reviewed and approved the final manuscript. Hee-Taik Kang are the guarantor of this work and, as such, had full access to all data in the study and takes responsibility for the integrity of the data and the accuracy of the data analysis.

Acknowledgement: This work was supported by the National Research Foundation of Korea (NRF) grant funded by the Korean government (MSIT) (Grant No. 2021R1G1A1006485)

Conflict Disclosure : No conflicts of interest

\section{References}

1. World Health Organization: Cardiovascular diseases (CVDs) fact sheets. 2021. URL: https://www.who.int/news-room/fact-sheets/detail/cardiovascular-diseases-(cvds)

2. Statistics Korea: Causes of Death Statistics in 2018. URL: http://kostat.go.kr/portal/eng/index.action.

3. Ormazabal V, Nair S, Elfeky O, Aguayo C, Salomon C, Zuñiga FA: Association between insulin resistance and the development of cardiovascular disease. Cardiovasc Diabetol 2018, 17(1):122.

4. Ausk KJ, Boyko EJ, loannou GN: Insulin resistance predicts mortality in nondiabetic individuals in the U.S. Diabetes Care 2010, 33(6):1179-1185. 
5. Pan K, Nelson RA, Wactawski-Wende J, Lee DJ, Manson JE, Aragaki AK, Mortimer JE, Phillips LS, Rohan T, Ho GYF et al: Insulin Resistance and Cancer-Specific and All-Cause Mortality in Postmenopausal Women: The Women's Health Initiative. J Natl Cancer Inst 2020, 112(2):170-178.

6. DeFronzo RA, Tobin JD, Andres R: Glucose clamp technique: a method for quantifying insulin secretion and resistance. Am J Physio/ 1979, 237(3):E214-223.

7. Fukushima M, Taniguchi A, Sakai M, Doi K, Nagasaka S, Tanaka H, Tokuyama K, Nakai Y: Homeostasis model assessment as a clinical index of insulin resistance. Comparison with the minimal model analysis. Diabetes Care 1999, 22(11):1911-1912.

8. Du T, Yuan G, Zhang M, Zhou X, Sun X, Yu X: Clinical usefulness of lipid ratios, visceral adiposity indicators, and the triglycerides and glucose index as risk markers of insulin resistance. Cardiovasc Diabetol 2014, 13:146.

9. Park B, Lee HS, Lee YJ: Triglyceride glucose (TyG) index as a predictor of incident type 2 diabetes among nonobese adults: a 12-year longitudinal study of the Korean Genome and Epidemiology Study cohort. Transl Res 2021, 228:42-51.

10. Lee SH, Kwon HS, Park YM, Ha HS, Jeong SH, Yang HK, Lee JH, Yim HW, Kang MI, Lee WC et al: Predicting the development of diabetes using the product of triglycerides and glucose: the Chungju Metabolic Disease Cohort (CMC) study. PLoS one 2014, 9(2):e90430.

11. Guerrero-Romero F, Simental-Mendía LE, González-Ortiz M, Martínez-Abundis E, Ramos-Zavala MG, Hernández-González SO, Jacques-Camarena O, Rodríguez-Morán M: The product of triglycerides and glucose, a simple measure of insulin sensitivity. Comparison with the euglycemic-hyperinsulinemic clamp. J Clin Endocrinol Metab 2010, 95(7):3347-3351.

12. Simental-Mendía LE, Rodríguez-Morán M, Guerrero-Romero F: The product of fasting glucose and triglycerides as surrogate for identifying insulin resistance in apparently healthy subjects. Metab Syndr Relat Disord 2008, 6(4):299-304.

13. Committee of Clinical Practice Guideline of the Korean Society of Lipid and Atherosclerosis (KSoLA): Korean Guidelines for the Management of Dyslipidemia 4th ed. 2018.

14. Korean Diabetes Association: Treatment guideline for diabetes, 6th edition. 2019.

15. American Diabetes Association: 2. Classification and Diagnosis of Diabetes: Standards of Medical Care in Diabetes-2020. Diabetes Care 2020, 43(Suppl 1):S14-s31.

16. Jung $\mathrm{CH}$, Choi KM: Impact of High-Carbohydrate Diet on Metabolic Parameters in Patients with Type 2 Diabetes. Nutrients 2017, 9(4):322.

17. Jung CH, Son JW, Kang S, Kim WJ, Kim HS, Kim HS, Seo M, Shin HJ, Lee SS, Jeong SJ et al: Diabetes Fact Sheets in Korea, 2020: An Appraisal of Current Status. Diabetes Metab J 2021, 45(1):110.

18. Kwon YJ, Lee JW, Kang HT: Secular Trends in Lipid Profiles in Korean Adults Based on the 20052015 KNHANES. Int J Environ Res Public Health 2019, 16(14):2555.

19. Lee SB, Kim MK, Kang S, Park K, Kim JH, Baik SJ, Nam JS, Ahn CW, Park JS: Triglyceride Glucose Index Is Superior to the Homeostasis Model Assessment of Insulin Resistance for Predicting 
Nonalcoholic Fatty Liver Disease in Korean Adults. Endocrinol Metab (Seoul, Korea) 2019, 34(2):179186.

20. Nam KW, Kwon HM, Jeong HY, Park JH, Kwon H, Jeong SM: High triglyceride-glucose index is associated with subclinical cerebral small vessel disease in a healthy population: a cross-sectional study. Cardiovasc Diabetol 2020, 19(1):53.

21. Cho YR, Ann SH, Won KB, Park GM, Kim YG, Yang DH, Kang JW, Lim TH, Kim HK, Choe J et al: Association between insulin resistance, hyperglycemia, and coronary artery disease according to the presence of diabetes. Sci Rep 2019, 9(1):6129.

22. Irace C, Carallo C, Scavelli FB, De Franceschi MS, Esposito T, Tripolino C, Gnasso A: Markers of insulin resistance and carotid atherosclerosis. A comparison of the homeostasis model assessment and triglyceride glucose index. Int J Clin Pract 2013, 67(7):665-672.

23. Lee SB, Ahn CW, Lee BK, Kang S, Nam JS, You JH, Kim MJ, Kim MK, Park JS: Association between triglyceride glucose index and arterial stiffness in Korean adults. Cardiovasc Diabetol 2018, 17(1):41.

24. Park B, Lee YJ, Lee HS, Jung DH: The triglyceride-glucose index predicts ischemic heart disease risk in Koreans: a prospective study using National Health Insurance Service data. Cardiovasc Diabetol 2020, 19(1):210.

25. Zhao Q, Zhang TY, Cheng YJ, Ma Y, Xu YK, Yang JQ, Zhou YJ: Triglyceride-Glucose Index as a Surrogate Marker of Insulin Resistance for Predicting Cardiovascular Outcomes in Nondiabetic Patients with Non-ST-Segment Elevation Acute Coronary Syndrome Undergoing Percutaneous Coronary Intervention. J Atheroscler Thromb 2020. Article in press

26. Guerrero-Romero F, Simental-Mendía LE, González-Ortiz M, Martínez-Abundis E, Ramos-Zavala MaG, Hernández-González SO, Jacques-Camarena O, Rodríguez-Morán M: The Product of Triglycerides and Glucose, a Simple Measure of Insulin Sensitivity. Comparison with the EuglycemicHyperinsulinemic Clamp. J Clin Endocrinol Metab 2010, 95(7):3347-3351.

27. Yu X, Wang L, Zhang W, Ming J, Jia A, Xu S, Li Q, Ji Q: Fasting triglycerides and glucose index is more suitable for the identification of metabolically unhealthy individuals in the Chinese adult population: A nationwide study. J Diabetes Investig 2019, 10(4):1050-1058.

\section{Tables}

Table 1. Baseline characteristics according to the triglyceride-glucose index quartile 


\begin{tabular}{|c|c|c|c|c|c|}
\hline Male & $\begin{array}{l}\mathrm{Q}_{1} \\
(<8.249)\end{array}$ & $\begin{array}{l}\mathrm{Q}_{2} \\
(8.249- \\
<8.614)\end{array}$ & $\begin{array}{l}\mathrm{Q}_{3} \\
(8.614- \\
<8.998)\end{array}$ & $\begin{array}{l}Q_{4} \\
(\geq 8.998)\end{array}$ & $\begin{array}{l}\mathrm{p}- \\
\text { value }\end{array}$ \\
\hline Number $(\mathrm{N})$ & 19,534 & 19,481 & 19,523 & 19,483 & N.A \\
\hline Age, years & $56.6 \pm 8.1$ & $56.2 \pm 7.9$ & $55.7 \pm 7.6$ & $54.7 \pm 6.9$ & $<0.001$ \\
\hline $\mathrm{BMI}, \mathrm{kg} / \mathrm{m}^{2}$ & $22.7 \pm 2.6$ & $23.5 \pm 2.6$ & $24.1 \pm 2.6$ & $24.8 \pm 2.6$ & $<0.001$ \\
\hline SBP, mmHg & $121.8 \pm 14.2$ & $124.0 \pm 14.2$ & $125.6 \pm 14.2$ & $127.4 \pm 14.2$ & $<0.001$ \\
\hline Glucose, mg/dL & $89.9 \pm 10.4$ & $93.5 \pm 10.6$ & $96.0 \pm 10.9$ & $99.4 \pm 11.2$ & $<0.001$ \\
\hline $\mathrm{TG}, \mathrm{mg} / \mathrm{dL}$ & $65.1 \pm 15.0$ & $100.6 \pm 15.2$ & $140.4 \pm 21.8$ & $248.7 \pm 98.3$ & $<0.001$ \\
\hline LDL-C, mg/dL & $112.7 \pm 31.6$ & $118.8 \pm 31.7$ & $120.3 \pm 32.6$ & $109.8 \pm 38.2$ & $<0.001$ \\
\hline TyG & $\begin{array}{l}7.945 \pm \\
0.247\end{array}$ & $8.438 \pm 0.105$ & $8.798 \pm 0.109$ & $\begin{array}{l}9.359 \pm \\
0.310\end{array}$ & $<0.001$ \\
\hline Ever smokers, N (\%) & $\begin{array}{l}11,437 \\
(58.5)\end{array}$ & $12,221(62.7)$ & $12,962(66.4)$ & $\begin{array}{l}13,800 \\
(70.8)\end{array}$ & $<0.001$ \\
\hline Drinking status, $\mathrm{N}(\%)$ & & & & & $<0.001$ \\
\hline Rare & $7,623(39.0)$ & 7,262 (37.3) & $6,681(34.2)$ & $5,708(29.3)$ & \\
\hline Sometimes & 7,964 (40.8) & $8,062(41.4)$ & $8,281(42.4)$ & $8,382(43.0)$ & \\
\hline Often & $3,947(20.2)$ & $4,157(21.3)$ & $4,561(23.4)$ & $5,393(27.7)$ & \\
\hline $\begin{array}{l}\text { Physical activity, } \mathrm{N} \\
\text { (\%) }\end{array}$ & & & & & $<0.001$ \\
\hline Rare & $3,954(20.2)$ & $4,069(20.9)$ & $4,068(20.8)$ & $4,208(21.6)$ & \\
\hline Sometimes & $8,607(44.1)$ & $9,077(46.6)$ & $9,267(47.5)$ & $9,452(48.5)$ & \\
\hline Regular & $6,973(35.7)$ & $6,335(32.5)$ & $6,188(31.7)$ & $5,823(29.9)$ & \\
\hline $\begin{array}{l}\text { Economic status, } \mathrm{N} \\
(\%)\end{array}$ & & & & & $<0.001$ \\
\hline Low & $3,172(16.2)$ & $3,025(15.5)$ & $2,909(14.9)$ & $2,871(14.7)$ & \\
\hline Middle & $5,890(30.2)$ & $5,994(30.8)$ & $5,742(29.4)$ & $5,924(30.4)$ & \\
\hline High & $\begin{array}{l}10,472 \\
(53.6)\end{array}$ & $10,462(53.7)$ & $10,872(55.7)$ & $\begin{array}{l}10,688 \\
(54.9)\end{array}$ & \\
\hline Female & $\begin{array}{l}Q_{1} \\
(<8.063)\end{array}$ & $\begin{array}{l}Q_{2} \\
(8.063- \\
<8.403)\end{array}$ & $\begin{array}{l}\mathrm{Q}_{3} \\
(8.403- \\
<8.752)\end{array}$ & $\begin{array}{l}Q_{4} \\
(\geq 8.752)\end{array}$ & $\begin{array}{l}\mathrm{p}- \\
\text { value }\end{array}$ \\
\hline
\end{tabular}




\begin{tabular}{|llllll|}
\hline Number (N) & 16,666 & 16,645 & 16,625 & 16,646 & N.A \\
\hline Age, years & $54.1 \pm 6.7$ & $55.6 \pm 7.5$ & $56.9 \pm 8.2$ & $58.3 \pm 8.6$ & $<0.001$ \\
\hline BMI, kg/m & $22.6 \pm 2.6$ & $23.2 \pm 2.7$ & $23.6 \pm 2.9$ & $24.3 \pm 2.9$ & $<0.001$ \\
\hline SBP, mmHg & $117.5 \pm 14.3$ & $119.8 \pm 14.7$ & $122.3 \pm 15.2$ & $124.4 \pm 15.3$ & $<0.001$ \\
\hline Glucose, mg/dL & $87.6 \pm 9.4$ & $91.1 \pm 9.5$ & $93.1 \pm 9.9$ & $96.1 \pm 10.6$ & $<0.001$ \\
\hline TG, mg/dL & $56.1 \pm 12.2$ & $84.5 \pm 11.8$ & $115.0 \pm 16.2$ & $194.4 \pm 73.8$ & $<0.001$ \\
\hline LDL-C, mg/dL & $116.7 \pm 30.9$ & $122.6 \pm 30.3$ & $125.5 \pm 33.7$ & $122.5 \pm 36.1$ & $<0.001$ \\
\hline TyG & $7.775 \pm$ & $8.240 \pm 0.097$ & $8.570 \pm 0.100$ & $9.086 \pm$ & $<0.001$ \\
\hline Ever smokers, N (\%) & $282(1.7)$ & $308(1.9)$ & $351(2.1)$ & $423(2.5)$ & $<0.001$ \\
\hline Drinking status, N (\%) & & & & & $<0.001$ \\
\hline Rare & 13,545 & $13,871(83.3)$ & $14,087(84.7)$ & 14,233 & \\
\hline Sometimes & $(81.3)$ & & & & \\
\hline Often & $2,671(16.0)$ & $2,336(14.0)$ & $2,133(12.8)$ & $1,988(11.9)$ & \\
\hline $\begin{array}{l}\text { Physical activity, N } \\
\text { (\%) }\end{array}$ & $450(2.7)$ & $438(2.6)$ & $405(2.4)$ & $425(2.6)$ & \\
\hline Rare & & & & & \\
\hline Sometimes & $7,259(43.6)$ & $7,130(42.8)$ & $7,078(42.6)$ & $6,999(42.0)$ & \\
\hline Regular & $5,073(30.4)$ & $4,800(28.8)$ & $4,606(27.7)$ & $4,466(26.8)$ & \\
\hline $\begin{array}{l}\text { Economic status, N } \\
\text { (\%) }\end{array}$ & & & & & \\
\hline Low & $4,334(26.0)$ & $4,715(28.3)$ & $4,941(29.7)$ & $5,181(31.1)$ & \\
\hline Middle & & & & & \\
\hline High & & & & & \\
\hline
\end{tabular}

Abbreviations: BMI, body mass index; SBP, systolic blood pressure; TG, triglycerides; LDL-C, low-density lipoprotein cholesterol; TyG, triglycerides-glucose index.

p-values are calculated from one-way ANOVA in case of continuous variables and from chi square test in case of categorical variables. 
Table 2. Cox-proportional hazards regression model for cardiovascular diseases, cerebrovascular diseases, cardio-cerebrovascular diseases, cardio-cerebrovascular diseases-related death, or all-cause death according to the TyG index quartile 


\begin{tabular}{|c|c|c|c|}
\hline Total & & Men & Women \\
\hline The primary outcomes & $\mathrm{Q}_{1}$ & Reference & Reference \\
\hline \multirow[t]{3}{*}{$\begin{array}{l}\text { (Cardio-cerebrovascular diseases and all-cause } \\
\text { mortality) }\end{array}$} & $\mathrm{Q}_{2}$ & $\begin{array}{l}1.063 \\
(0.982-1.152)\end{array}$ & $1.099(0.986-1.226)$ \\
\hline & $\mathrm{Q}_{3}$ & $\begin{array}{l}1.112 \\
(1.026-1.206)\end{array}$ & $1.049(0.941-1.169)$ \\
\hline & $\mathrm{Q}_{4}$ & $\begin{array}{l}1.153 \\
(1.060-1.254)\end{array}$ & $1.069(0.960-1.190)$ \\
\hline Subgroups & & Men & Women \\
\hline \multirow[t]{4}{*}{ Cardiovascular diseases } & $\mathrm{Q}_{1}$ & Reference & Reference \\
\hline & $\mathrm{Q}_{2}$ & $\begin{array}{l}1.117 \\
(0.971-1.285)\end{array}$ & $1.239(1.018-1.509)$ \\
\hline & $\mathrm{Q}_{3}$ & $\begin{array}{l}1.191 \\
(1.036-1.369)\end{array}$ & $1.188(0.976-1.446)$ \\
\hline & $\mathrm{Q}_{4}$ & $\begin{array}{l}1.237 \\
(1.071-1.427)\end{array}$ & $1.248(1.027-1.517)$ \\
\hline \multirow[t]{4}{*}{ Cerebrovascular diseases } & $Q_{1}$ & Reference & Reference \\
\hline & $\mathrm{Q}_{2}$ & $\begin{array}{l}1.020 \\
(0.900-1.156)\end{array}$ & $0.988(0.853-1.144)$ \\
\hline & $\mathrm{Q}_{3}$ & $\begin{array}{l}1.073 \\
(0.946-1.217)\end{array}$ & $1.011(0.876-1.168)$ \\
\hline & $\mathrm{Q}_{4}$ & $\begin{array}{l}1.128 \\
(0.991-1.285)\end{array}$ & $1.009(0.874-1.165)$ \\
\hline \multirow[t]{4}{*}{ Cardio-cerebrovascular diseases-related deaths } & $Q_{1}$ & Reference & Reference \\
\hline & $\mathrm{Q}_{2}$ & $\begin{array}{l}1.231 \\
(0.778-1.947)\end{array}$ & $0.907(0.405-2.031)$ \\
\hline & $\mathrm{Q}_{3}$ & $\begin{array}{l}1.157 \\
(0.714-1.876)\end{array}$ & $1.452(0.708-2.977)$ \\
\hline & $\mathrm{Q}_{4}$ & $\begin{array}{l}1.168 \\
(0.698-1.954)\end{array}$ & $1.252(0.606-2.586)$ \\
\hline \multirow[t]{4}{*}{ All-cause deaths } & $Q_{1}$ & Reference & Reference \\
\hline & $\mathrm{Q}_{2}$ & $\begin{array}{l}1.046 \\
(0.910-1.204)\end{array}$ & $1.216(0.936-1.580)$ \\
\hline & $\mathrm{Q}_{3}$ & $\begin{array}{l}1.103 \\
(0.954-1.276)\end{array}$ & $1.010(0.776-1.314)$ \\
\hline & & 1.063 & $0.992(0.765-1.286)$ \\
\hline
\end{tabular}


Adjusted for age, smoking status (ever and never smokers), drinking status (rare, sometimes, and often) and physical activity (rare, sometimes, and regular), body mass index, systolic blood pressure, LDL-C, and economic status (low, middle, and high)

\section{Figures}

\section{Random sample $(\mathrm{n}=362,285)$}

From health screening examinee between 2009 and 2010

\section{Exclude candidates $(n=217,682)$}

(1) who died between 2009 and $2011(\mathrm{n}=3,644)$

(2) who had a fasting blood glucose level $\geq 126 \mathrm{mg} / \mathrm{dL}$ between 2002 and $2010(\mathrm{n}=64,448)$ or

(3) who were prescribed anti-diabetic drugs between 2002 and $2010(n=9,695)$ or

(4) who were diagnosed with diabetes between 2002 and 2010 (ICD-10 code: E10-E14) $(n=20,580)$ or

(5) who were diagnosed with neoplasm (ICD-10 code: C00-C97 or D00-D04, D08-D09) $(n=24,789)$ or cardio/cerebrovascular disease (ICD-10 code: I20-I25, I60-I69) $(n=55,095)$ between 2002 and 2011 or

(6) who had any history of diabetes, heart disease, or cardio/cerebrovascular disease according to the self-reported questionnaires of the health examination between 2002 and $2010(n=2,686)$ or

(7) who were prescribed anti-dyslipidemic agents (statin, omega-3 fatty acid, niacin, or cholesterol sequestrants) between 2002 and $2010(n=30,615)$ or

(8) Who had incomplete data for the confounders $(n=6,036)$

(9) Who had total study duration of 30 days or less $(n=94)$

\section{Figure 1}


2A-1) Men

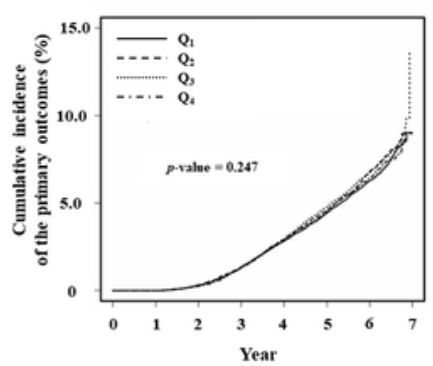

$\begin{array}{llllllll}\mathrm{Q}_{1} & 0.01 & 0.33 & 1.35 & 2.84 & 4.44 & 6.30 & 9.03\end{array}$

$\begin{array}{lllllllll}Q_{2} & 0.01 & 0.30 & 1.35 & 2.93 & 4.61 & 6.84 & 8.98\end{array}$

$\begin{array}{llllllllll}\text { Q } & & 0.01 & 0.33 & 1.32 & 2.95 & 4.79 & 6.77 & 13.5\end{array}$

$\begin{array}{llllllllll}Q_{4} & 0.01 & 0.25 & 1.33 & 2.86 & 4.52 & 6.46 & 8.98\end{array}$
2A-2) Women

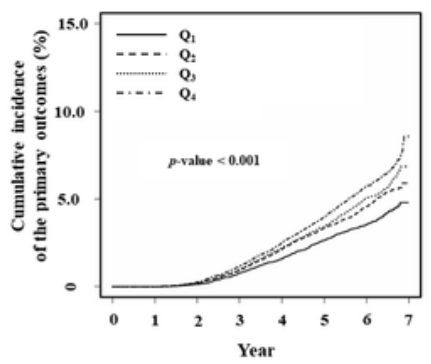

$\begin{array}{llllllll}0.01 & 0.14 & 0.78 & 1.59 & 2.65 & 3.57 & 4.80\end{array}$ $\begin{array}{lllllll}0.01 & 0.22 & 0.94 & 2.13 & 3.36 & 4.58 & 5.90\end{array}$ $\begin{array}{lllllll}0.01 & 0.20 & 0.98 & 2.22 & 3.42 & 5.06 & 6.85\end{array}$ $\begin{array}{lllllll}0.01 & 0.27 & 1.18 & 2.53 & 4.00 & 5.77 & 8.57\end{array}$

Estimated cumulative incidence of the primary outcomes

2B-1) Men

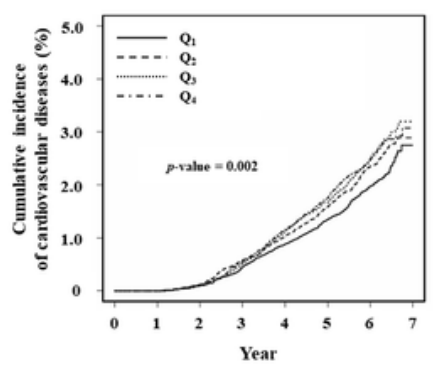

$\begin{array}{lllllll}0.01 & 0.10 & 0.45 & 0.88 & 1.35 & 1.99 & 2.75\end{array}$ $\begin{array}{llllllll}0.01 & 0.12 & 0.58 & 1.01 & 1.59 & 2.37 & 2.89\end{array}$ $\begin{array}{lllllll}0.01 & 0.12 & 0.52 & 1.12 & 1.68 & 2.51 & 3.20\end{array}$ $\begin{array}{lllllll}0.01 & 0.11 & 0.56 & 1.16 & 1.75 & 2.48 & 3.07\end{array}$
2B-2) Women

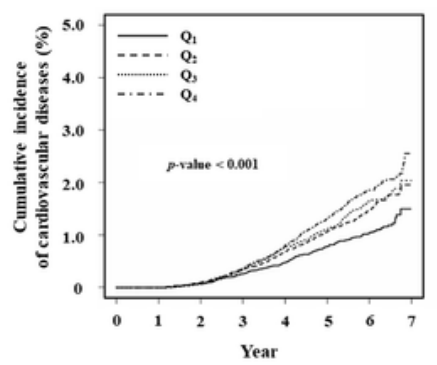

$\begin{array}{llllllll}0.01 & 0.08 & 0.26 & 0.48 & 0.78 & 1.04 & 1.50\end{array}$ $\begin{array}{llllllll}0.01 & 0.10 & 0.36 & 0.69 & 1.08 & 1.49 & 1.96\end{array}$ $\begin{array}{lllllll}0.01 & 0.07 & 0.33 & 0.78 & 1.11 & 1.65 & 2.04\end{array}$ $\begin{array}{llllllll}0.01 & 0.09 & 0.37 & 0.81 & 1.33 & 1.87 & 2.55\end{array}$

Estimated cumulative incidence of cardiovascular diseases

2C-1) Men

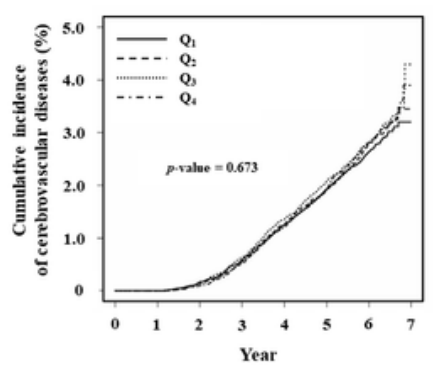

$\begin{array}{lllllllll}\mathrm{Q}_{1} & & 0.01 & 0.17 & 0.59 & 1.26 & 1.93 & 2.65 & 3.20\end{array}$

$\begin{array}{lllllllll}Q_{2} & 0.01 & 0.13 & 0.53 & 1.25 & 1.91 & 2.79 & 3.45\end{array}$

$\begin{array}{llllllll}\text { Q. } & 0.01 & 0.17 & 0.64 & 1.36 & 2.09 & 2.83 & 4.29\end{array}$

$\begin{array}{lllllllll}Q_{4} & 0.01 & 0.10 & 0.56 & 1.22 & 1.95 & 2.83 & 3.90\end{array}$
2C-2) Women

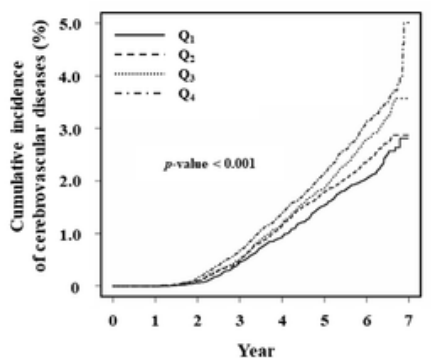

$\begin{array}{llllllll}0.01 & 0.07 & 0.46 & 0.93 & 1.55 & 2.05 & 2.81\end{array}$ $\begin{array}{llllllll}0.01 & 0.11 & 0.50 & 1.16 & 1.80 & 2.38 & 2.87\end{array}$ $\begin{array}{lllllll}0.01 & 0.10 & 0.52 & 1.18 & 1.86 & 2.81 & 3.57\end{array}$ $\begin{array}{llllllll}0.01 & 0.17 & 0.69 & 1.40 & 2.15 & 3.15 & 5.01\end{array}$

Estimated cumulative incidence of cerebrovascular diseases

\section{Figure 2}

The estimated cumulative incidence of each outcome A) The primary outcomes (cardio-cerebrovascular diseases and all-cause mortality) B) Cardiovascular diseases, only C) Cerebrovascular diseases, only All p-values are from log-rank tests.

\section{Supplementary Files}


This is a list of supplementary files associated with this preprint. Click to download.

- SupplTable.docx 